\title{
Feedback from the Fifth European Bioanalysis Forum Young Scientist Symposium
}

\author{
Michele Protti ${ }^{1}$, Toon Babylon ${ }^{2}$, Lisa Delahaye ${ }^{3}$, Jörg Faber ${ }^{4}$, Christopher Fox ${ }^{5}$, Farjana \\ Mahammed $^{6}$, Laura Mercolini ${ }^{1}$, Tessa McDonald ${ }^{7}$, Mathias Salger $^{8}$, Alexandra Vantcheva ${ }^{9}$ \\ \& Philip Timmerman*,10 \\ ${ }^{1}$ University of Bologna, 40126 Bologna, Italy \\ ${ }^{2}$ UCB Biopharma, 1420 Braine-I'Alleud, Belgium \\ ${ }^{3}$ Ghent University, 9000 Ghent, Belgium \\ ${ }^{4}$ A\&M Labor, 50126 Bergheim, Germany \\ ${ }^{5}$ AstraZeneca, Cambridge, CB4 OWG, UK \\ ${ }^{6}$ GlaxoSmithKline, Ware, SG12 ODP, UK \\ ${ }^{7}$ York Bioanalytical Solutions, York, YO26 6QR, UK \\ ${ }^{8}$ Nuvisan, 85567 Grafing, Germany \\ ${ }^{9}$ Comac Medical, Sofia, Bulgaria \\ ${ }^{10}$ European Bioanalysis Forum vzw (EBF), 1000 Brussels, Belgium \\ *Author for correspondence: Tel. +32 4799101 32; chair@e-b-f.eu
}

Since 2014, the European Bioanalysis Forum (EBF) organizes a Young Scientist Symposium. The meeting format was created to provide development opportunities for young scientists to engage in international discussions. Creating a peer community of young scientists has been a proven recipe to lower the threshold and promote engagement in this community of young talents. At the same time, the meetings are aimed at stimulating collaboration between the EBF and academia. This manuscript summarizes the discussions at the Fifth EBF Young Scientist Symposium, held in Bologna (Italy) between 20 and 21 March 2019.

First draft submitted: 28 June 2019; Accepted for publication: 14 August 2019; Published online: 12 September 2019

The Fifth European Bioanalysis Forum (EBF) Young Scientist Symposium (YSS) was organized in an evocative historical building headed by Alma Mater Studiorum - University of Bologna, in order to strengthen the successful liaison between industry and academia. In fact, this encases the spirit of the EBF YSS meeting; able to create a sandbox to generate new networks to flow ideas, share points of view and light the fuse of new and prosperous collaborations.

The 2-day program was divided into two complementary formats in a successful formula. During the first day, around 70 attendees met in round tables focused on different hot topics of bioanalysis led by the organizing committee $(\mathrm{OC})$ members' expertise. The second day was organized as a plenary meeting featuring 21 oral communications pivoting around four main themes and converging into panel discussions, 24 posters exhibited during dedicated sessions, of which ten were presented as short poster pitches and competed for the best poster award.

\section{Science cafe}

On day 1 , the symposium kicked off with a dynamic science cafe in which the young scientist community had the opportunity to network and exchange ideas via round table discussions. All attendees were divided over eight tables, each being moderated by a member of the OC. The OC prepared a set of questions, identified from the release of the 2018 US FDA Bioanalytical Method Validation (BMV) Guidance [1], focusing on four different themes:

- Biomarkers/endogenous compounds;

- Fit-for-purpose (FFP);

- Dried blood spot (DBS)/microsampling; 
- Incurred sample reanalysis (ISR).

An open discussion among peers should help with the individual's understanding of the importance of the updates, and the importance of harmonization across the bioanalytical community.

\section{Theme 1: Biomarkers/endogenous compounds}

As biomarkers are potentially endogenously present in the analyzed matrix, discussions started with the limitations of such assays and how to overcome them. The most important limitation identified was to find a suitable blank matrix free of analyte. The attendees shared experiences and ideas such as using the same matrix but from a different species or gender, over extracting the analyte from the matrix, for example, by charcoal stripping or washing blood cell fraction, to using commercially available or self-made artificial surrogate matrices. However, it should be demonstrated that the chosen matrix mimics the original matrix as well as possible, for example, by parallelism experiments, as stipulated in the 2018 FDA BMV.

The discussions further led to the question of what the attendees think of the FDA's expectation to validate biomarker assays based on the experiments and acceptance criteria for pharmacokinetics (PK) assays and to fully validate biomarker assays in case of pivotal studies. All agreed that this depended entirely on the context of use of the biomarker data and what the requirements of the sponsor/stakeholder are. In addition to understanding the mode of action and biology, it was agreed that a thorough understanding of the physiochemical and biochemical properties of the biomarker are needed before embarking on establishing a method.

A productive debate developed on whether or not a guidance documents for biomarkers is needed. The use of the same acceptance criteria as for PK assays was seen to strongly depend on the context of use of the biomarker data, as the needed precision and accuracy of a PK assay does not always apply to biomarkers. As the requirements regarding precision and accuracy of biomarker assays often cannot be decided on by the analyst, a close interdisciplinary teamwork between all stakeholders (e.g., biologists, biochemists and analysts) was seen as crucial.

The delegates saw no need for guidance by a regulatory agency but wished to have a harmonization of biomarker validations. Thus, all delegates were reminded on the EBF recommendation paper for the analysis of biomarkers [2]. The young scientists also appreciated the announcement of a Focus Workshop on biomarker assay validation by the EBF in the fall.

\section{Theme 2: FFP}

The recent inclusion of the FFP concept in the 2018 FDA BMV Guidance creates the opportunity to apply a FFP approach for validation of methods used in a regulated bioanalytical environment.

In the second session of the science cafe evening, the delegates first discussed their own experience with FFP validations. Where most of them have only performed regular, full validations, there were some examples given of FFP validations in the exploratory and preclinical stages of early drug development. Although their experience with FFP was limited; all delegates recognized the added value that FFP offers and the savings that can be made in terms of time and cost. Furthermore, the opinion of the delegates was that even though in some instances an additional, full validation may be necessary, the benefits of a FFP approach in the early drug development stage will still outweigh the additional work that should be performed for a full validation in a later stage.

A point of discussion between the delegates was who is finally deciding on the level of validation for a certain method. In summary, they agreed that the sponsor is most likely to have the final decision about the level of validation because they have the knowledge of where and how the data will be used. Nevertheless, pharma representatives indicated that they would welcome more discussion on this issue with the contract research organization (CRO) scientists. The delegates acknowledged that open communication between all parties is key in the discussion around the desired level of validation and, again, that all stakeholders should be involved.

As for biomarkers, the delegates saw no need for additional regulation on how to perform a FFP validation, however, harmonization between guidelines and a common understanding of the studies in scope for FFP by different regulatory agencies and industry would promote a broader acceptance of the FFP approach in the regulated bioanalytical environment.

\section{Theme 3: DBS/microsampling}

Technologies for collecting microvolumes of blood/plasma/serum for the accurate determination of circulating concentrations of therapeutic drugs, metabolites and biomarkers in preclinical and clinical studies continue to 
impact how we look at bioanalysis. Delegates discussed their thoughts on not only the positives aspects and promising potentials but also the limitations and drawbacks.

The main issue faced by DBS sampling continues to be the effect of the hematocrit [3], leading to volumetric bias and sample inhomogeneity. With alternative technologies, for example, volumetric absorptive microsampling (VAMS) [4] and the hemaPEN2 [5], already being researched or adopted by companies in industry, the technology continues to show potential and deliver on promise. With the implementation of such devices opening the doors for patient convenient sampling, whether that be at home or in remote areas, will come the need for additional parameters to be included in validation. Delegates noted ambient storage and handling temperature, homogeneity of sample spotting, analyte-dependent stability, reproducibility including ISR, as well as extraction solvent and recovery will need to be considered and addressed.

The 2018 FDA BMV states that when using DBS in regulated studies, correlative studies with traditional sampling should be conducted during drug development. At the current time, as referenced in the ICH M3 (R2) Q\&A [6], it is necessary to conduct a clinical PK bridging study with both plasma and DBS collected to confirm correlation. Discussions voiced the need for clear and shared guidelines on what percentage of samples in the study needs to be in both wet and dry matrix, whether too many requirements will hinder the technology, and will all new compounds require correlative studies.

The acceptance of new microsampling technologies by regulatory authorities is key. With the generation of reliable data alongside a widespread use within the frameworks where such technologies are more promising (e.g., pediatrics and home sampling), the bioanalytical (BA) community can contribute to their development and adoption. There was agreement from the young scientists to be the voice for change and deliver the message within their companies to overall break the mould from the classical sampling methods, and move towards the future, where microsampling will become a standard.

The EBF and their current research work into microsampling [7-9] have been recognized and valued by all delegates as one of the main sources of knowledge and insights.

\section{Theme 4: ISR}

Theme 4 of the science cafe revolved around the importance of ISR and its recent changes in the 2018 FDA BMV. An introduction to the principles of ISR demonstrated that most attendees are comfortable with the function of ISR as an additional validation parameter that is performed on incurred samples. It was also discussed how ISR forms part of the validation, rather than part of the sample analysis and submission.

When debating the times where ISR is required, delegates felt that further clarity is still required surrounding the use of 'pivotal' as a descriptor for a study requiring ISR. Hence, ISR is probably conducted in more studies than actually needed regarding current guidelines. Delegates agreed that it is essential to consider carefully when ISR testing is required to avoid unnecessary analyses. Concerning sample selection, a consensus was reached that selecting samples to satisfy the $\mathrm{C}_{\max }$ and elimination phase requirement of the 2018 FDA BMV, goes hand in hand with a scientific desire to validate results across as many unique subjects and time points as possible.

Delegates who have experience of performing ISR noted the low incidence of failure, and the investigation in to the root cause being planned in a bespoke manner. Where young scientists have performed root cause analysis it has been identified as due to insufficient sample stability, analyst error or issues with sample homogeneity. These findings mimic those presented in previous EBF papers surrounding ISR [10].

\section{Podium presentations}

\section{Session 1: Let us continue small}

The first session of the meeting brought the focus of microsampling to the attention of the delegates. An introduction by Farjana Mahammed (GlaxoSmithKline) highlighted the journey of microsampling, from the 1990s where DBS was first introduced, to the current day where alternative sampling approaches have the potential to allow a patient to take a small sample, anywhere, at any time, benefitting patient-centered care.

An array of four presentations was followed by a panel discussion on the topic of 'Future Avenues for Small Volume Sampling in the Clinic'.

The opening presentation was delivered by Lisa Delahaye (U-Ghent) focusing on VAMS as an alternative tool for adduct-based monitoring of paracetamol toxicity. Quantitating on APAP-Cys as an alternative biomarker for paracetamol toxicity, Lisa validated a specific VAMS-based approach. 
Michele Protti (Unibo) spoke about the bioanalytical outcomes from a 4-year workplace alcohol consumption monitoring project. His work addressed the use of DBS and dried urine spots biological samples coupled to instrumental analytical methodologies based on liquid chromatography and capillary electrophoresis for assessment of ethyl glucoronide and ethyl sulfate. The penultimate presentation by Matthijs Pijnappel (Recipharm) focused on a head-to-head comparison of DBS and traditional plasma PK sampling in a clinical trial. Matthijs showed good reproducibility of the PK data obtained, in accordance with ISR bioanalytical data acceptance criteria from EMA [11] and 2018 FDA BMV.

To finish, Ian Dawson (Covance) presented on the considerations for the use of VAMS devices for a clinical bioanalytical assay. His talk evaluated variability of wicking volume as well as the results of internal standard loading pre- and postsampling.

A lively debate followed the core presentations between representatives of drug developers (sponsors), CROs, academia and pharma companies. This provided confirmation that all the players involved in miniaturized and alternative sampling have moved into an exciting time in bioanalytical research. In the past years, we have seen a greater commitment to microsampling from several large companies, as well as from our regulators and from the whole BA community. Limitations for large scale onboarding could be from a lack of evolution in technologies as well as a lack of sufficient interest from the clinical field. However, thanks to advances in science, it is now possible to generate a large amount of data from a small amount of biological fluid. With this being a known, the EBF and their current research work into microsampling, through an industry consortium, will be most significant to the continued acceptance and application.

\section{Session 2: From applications to lunch}

Within this session we received excellent abstracts from presenters to examine how the current challenges associated with established technologies could be overcome and how their experiences as young scientists had allowed them to push the boundaries. Jessica Knox (Charles River) opened the session on in vitro bioanalysis and demonstrated a faster and more cost effective way of getting topical treatments to the market. Chris Nortcliffe (Sciex) continued with a presentation about the increased sensitivity possible when using microflow trap-elute methodologies for the detection of antibody-drug conjugates and monoclonal antibodies. Utilizing this system allowed for between 8and 60-times increase in S/N at the LLOQ. Quentin Enjalbert (Anaquant) spoke about simplifying the process by which proteins are identified and quantified by using a novel technique based on relative quantification. He outlined a technique that identified and quantified 361 discrete proteins within a sample.

Sophia Christou (LGC) highlighted the importance of therapeutic drug monitoring technologies and the innovative ways CROs are meeting the needs of supporting emerging therapeutic drug monitoring requirements. To close out the session, Annelies Cannaert (Ghent University) presented on a cell-based method by which novel psychoactive substances can be detected independently of the structure but based on their activity.

In summary, this session showed the ingenuity of young scientists as they tackle the bioanalytical challenges of today and tomorrow.

\section{Session 3: Platform 93/4}

For the third session there was a focus on laboratory platforms used in bioanalysis; five speakers presented on various platforms used in a range of immunoassay techniques.

Katja Zeiser (Nuvisan) opened the session with a presentation outlining the key factors to consider overcoming the issues in mesoscale discovery, an analytical platform (MSD) assay development described in two case studies.

Toon Babylon (UCB Biopharma) spoke about bridging a classic serum PK assay to cerebrospinal fluid, which showed the pitfalls of surrogate matrices and the benefits of automation.

Sarah Collomb (Novimmune) compared the Gyrolab and mesoscale discovery platforms for antidrug antibody testing, demonstrating that Gyrolab is a viable alternative with potential of a greater throughput.

Christopher Fox (AstraZeneca) compared the Gyrolab and the Ella instruments for a biomarker assay and demonstrated that due to antibody differences in proprietary kits, measurement of the same biomarker can be variable between platforms.

Florian Neff (F Hoffmann - La Roche) closed the session with a presentation on the Ella for preclinical drug quantification and antidrug antibody/immunogenicity, showing consistent results in comparison with ELISA platform. 
In summary, this session provided an insight into a variety of challenges and solutions by implementing automation into bioanalytical procedures and displayed the increasing interest of the young scientist into new platforms and methodologies.

\section{Session 4: Do not leave yet, there is more}

In the closing session of the symposium, four presenters focused on critical aspects during method development and validation.

In the first presentation, Jenny Baker (LGC) presented Rapid Equilibrium Dialysis, a device to cope with the issue of plasma protein binding.

Next, Alexandra Vantcheva (Comac Medical) highlighted the importance of using an appropriate internal standard for LC-MS/MS methods. More specifically, she presented a case example of a recombinant polypeptide where a stably labeled isotope performed better as internal standard compared with a structural analog.

Matthew Sciscione (Syneos Health) demonstrated in his presentation how LC-MS/MS can be an important tool to assure the quality and to improve the consistency of critical reagents used in ligand-binding assays.

The last presentation of the day was given by Laure Queyrel (Envigo) who suggested a simplified and FFP immunogenicity strategy to support preclinical studies. A special focus was given to the difference between two approaches for cut-point determination during assay validation.

In conclusion, the presenters in this session gave some interesting examples of how different aspects in method development and validation can have a large impact on the use and performance of a bioanalytical assay.

\section{Poster session}

The poster presentations provided samples of innovative research, promising preliminary results and focused original applications dealing with the many facets of bioanalysis and composing a suggestive kaleidoscope of good science. Among these, the best poster award was given to Sergio Menta (Celerion) for a research work regarding a novel trap-and-elute LC-MS/MS method to quantify acyl ghrelin and des-acyl ghrelin in human plasma.

\section{Conclusion}

Once again, for this fifth edition of the EBF YSS the promises have been kept, producing an event of high scientific value thanks to both the great commitment put by the $\mathrm{OC}$ and the high degree of participation and interaction between attendees.

During the science cafe, discussions among peers centered on regulatory aspects of bioanalysis and ensured that the young scientists involved gave rise to fruitful considerations in a mutual exchange of points of view. This foresaw how the attendees, as future bioanalytical leaders who will have to deal with such guidelines, will integrate them into their scientific projects in a constructively critical manner.

On the other hand, the plenary session was rich in content with a high scientific value. The lectures addressed the main current bioanalytical challenges by proposing ingenious and cutting-edge solutions together with the panel discussions, characterized by a high degree of interaction.

In the wake of this edition's success, a new YSS OC is already at work to design and develop the sixth EBF YSS, pivoting on all the factors positively contributing to this edition, in a constant and continuous improvement. This meeting will be held in the same venue between 19 and 20 March 2020. The desire is to guarantee a fertile ground for young bioanalytical scientists, where they can share their experience and ideas in mutual enrichment for themselves and their organizations.

\section{Acknowledgments}

The authors want to thank all presenters for their contribution to the Fifth Young Scientist Symposium.

\section{Disclaimer}

The views and conclusion presented in this paper are those of the European Bioanalysis Forum and do not necessarily reflect the representative affiliation or company's position on the subject. 


\section{Financial \& competing interests disclosure}

The authors have no relevant affiliations or financial involvement with any organization or entity with a financial interest in or financial conflict with the subject matter or materials discussed in the manuscript. This includes employment, consultancies, honoraria, stock ownership or options, expert testimony, grants or patens received or pending, or royalties.

No writing assistance was utilized in the production of this manuscript.

\section{References}

1. US FDA. Guidance for industry: bioanalytical method validation (2018). www.fda.gov/downloads/drugs/guidances/ucm070107.Pdf

2. Timmerman P, Herlig C, Stoellner D et al. European Bioanalysis Forum recommendation on method establishment and bioanalysis of biomarkers in support of drug development. Bioanalysis 4(15), 1883-1894 (2012).

3. De Vries R, Barfield M, van de Merbel N et al. The effect of hematocrit on bioanalysis of DBS: results from the EBF DBS-microsampling consortium, Bioanalysis 5(17), 2147-2160. (2013)

4. Neoteryx. Smarter, simpler blood collection (2019). www.neoteryx.com/

5. Trajan. hemaPEN (2019). www.trajanscimed.com/pages/hemapen

6. EMA. ICH M3 (R2) Q\&A (2012). www.ema.europa.eu/en/documents/other/international-conference-harmonisation-technical-requir ements-registration-pharmaceuticals-human-use_en.pdf

7. Timmerman P, White S, Cobb Z et al. Update of the EBF recommendation for the use of DBS in regulated bioanalysis integrating the conclusions from the EBF DBS-microsampling consortium. Bioanalysis 5(17), 2129-2136 (2013).

8. Cobb Z, Rohde M, Love I et al. Feedback from the EBF liquid microsampling consortium: capillary liquid microsampling and homogeneity of the resultant samples. Bioanalysis 11(6), 525-532 (2019).

9. Hawthorne G, Dillen L, Barfield M et al. Feedback from the EBF liquid micro sampling consortium: microsampling: assessing accuracy and precision of hand held pipettes and capillaries. Bioanalysis 11(6), 533-542 (2019).

10. Kall MA, Michi M, van der Strate B et al. Incurred sample reproducibility - 10 years of experiences: views and recommendations from the European Bioanalytical Forum. Bioanalysis 10(21), 1723-1732 (2018)

11. EMA. Guideline on bioanalytical method validation. EMEA/CHMP/EWP/192217/2009

(2011). www.ema.europa.eu/documents/scientific-guideline/guideline-bioanalytical-method-validationen.pdf 